\title{
On the work hardening of titanium: new insights from nanoindentation
}

\author{
Arnas Fitzner $^{1}$ (1), Jack Palmer ${ }^{1}$ (D), Ben Gardner $^{1}$ (D), Matthew Thomas $^{2}$ (D), Michael Preuss ${ }^{1}$ (D), and \\ Joao Quinta da Fonseca ${ }^{1, *}$ (i) \\ ${ }^{1}$ The School of Materials, The University of Manchester, Oxford Road, Manchester M13 9PL, UK \\ ${ }^{2}$ Timet, Wilton, PO Box 704, Birmingham B6 7UR, UK
}

Received: 17 August 2018

Accepted: 6 February 2019

Published online:

21 February 2019

(C) The Author(s) 2019

\begin{abstract}
Nanoindentation was used to probe the local slip resistance in CP-Ti deformed in compression to different extents. Changes in hardness in the deformed grains and twins were compared with the change in flow stress measured during deformation, with the aim to elucidate the relative contribution of slip and twinning to the work hardening of Ti alloys. The hardness values were calibrated with measurements on binary $\mathrm{Ti}-\mathrm{Al}$ alloys. The hardness increased only slightly with deformation and cannot explain the observed work hardening. Although twinned regions were found to be harder than the parent grains, this increase was found to be small once the effect of crystal orientation was accounted for. The increase in hardness in the twins was slightly higher for compressive twins than for tensile twins. It is proposed that this modest hardness increase in the twins is more consistent with the presence of twinning stresses than with a change in the local flow stress caused by dislocation interactions. The implications of these findings to the work hardening of $\mathrm{CP}$ titanium are discussed.
\end{abstract}

\section{Introduction}

The plastic deformation of commercial pure $\alpha$-titanium at room temperature is strongly anisotropic at the single crystal level [1] because slip parallel to the closely packed planes of its HCP lattice is much easier than along other directions. $\alpha$-titanium also twins relatively easily in a number of different ways. Unlike slip, twinning depends not just on the resolved shear stress but also on its sign. In the polycrystalline material, the deformation behaviour is controlled by the relative activation of the different deformation mechanisms and their interaction, which will therefore depend strongly on the crystallographic texture and deformation mode and is difficult to predict.

As a consequence of the activity of multiple, interacting deformation mechanisms, the hardening behaviour of $\alpha$-titanium is anisotropic, path dependent, asymmetric and shows pronounced reverseloading effects [2-5]. During uniaxial loading, the initial rate of hardening is always higher in

Address correspondence to E-mail: joao.fonseca@manchester.ac.uk 
compression than in tension irrespective of texture [2-4] and a characteristic increase in work hardening rate is seen at effective strains of about 0.04 in uniaxial compression along ND and slightly later (0.05) in plane strain [3]. During plane strain compression (PSC), the hardening behaviour depends on the loading direction with respect of the starting texture [5]. The work hardening rate increases with strain rate and decreases with temperature [6].

Although it is generally accepted that this hardening behaviour is dominated by twinning, there is disagreement as to the reason why. Some researchers propose that twinning causes hardening because it changes the defect structure and increases the local flow stress within the twin. Salem and co-workers have proposed that twinning causes hardening by the Basinski effect, whereby dislocations are rendered sessile by twinning [2, 7], and more recently, a mechanism has been suggested whereby dislocations are incorporated in the twin volume during twin growth, leading to very strong latent hardening in the twin [8]. Others claim instead that twinning causes hardening primarily because it modifies the microstructure by changing the texture and by reducing the slip length. For example, Battainni and co-workers compared results from PSC tests along different directions and suggested that the difference in hardening behaviour could be explained simply by reorientation hardening caused by tensile twinning, balanced in some cases by orientation softening caused by compression twining [5].

These details are important because they determine which mechanisms should be considered in crystal plasticity models. For example, in the work by Salem et al. [9] the mechanisms proposed in earlier work $[2,7]$ are used to justify changing the slip resistance after twinning but reorientation due to twinning is not accounted for explicitly and hence the corresponding hardening (or softening) effects are not considered. Other models account for reorientation but also include a Hall-Petch term to account for a decrease in slip length [10]. More recently, Oppedal et al. [11] showed that the Hall-Petch contribution could be ignored provided the latent hardening in the twinned volumes was made significantly higher than that in the parent grain, which was explained by a dislocation transmutation mechanism [8].

One of the difficulties is that the experimental data on the effects of twinning on work hardening are contradictory. Nanoindentation measurements by
Salem et al. [7] showed that the twinned volume is much harder than the matrix, lending support to the idea that the twinning process increases the flow stress of the material. This has been supported by crystal plasticity models, which have shown that changes in texture caused by twinning reorientation can only explain some of the observed hardening. Furthermore, the work hardening is higher in compression than in tension, despite the fact that compression twinning should induce texture softening by reorientation. However, this is in conflict with results from temperature reversal experiments in Ti [6] and also in $\mathrm{Zr}$ [12, 13], which showed that although twinning is more profuse at $77 \mathrm{~K}$, material compressed at cryogenic temperatures has a lower flow stress once reloaded at RT than an equivalent sample previously deformed at RT, supporting the idea of orientation softening and contradicting the idea of a Hall-Petch effect and of a twin-induced change in slip resistance. Furthermore, recent in situ TEM work which has shown that although twining creates defects that interact with pre-existing dislocations, the resultant dislocation in the twin is mobile and not sessile as would be expected due to the Basinski mechanism [14].

In the work presented here, nanoindentation was used to study the local slip resistance of deformed commercial purity (CP) Ti. These measurements complement those by Salem and co-workers [7], which to date provide the strongest direct evidence for hardening via a dislocation-twin interactionbased mechanism. The interpretation of nanoindentation measurements is not easy in Ti because, due to its anisotropy, the nanohardness varies significantly with indentation orientation, for example, see [15], and slip activity is complex during nanoindentation, making it difficult to relate differences in nanohardness to changes in the flow stress in individual slip systems. In the measurements shown here, the effects of orientation on measured hardness were carefully accounted for using electron backscatter diffraction (EBSD) mapping. In addition, the values measured were calibrated using orientation-corrected measurements on a series of binary Ti-Al alloys with known proof stresses. The nanohardness was measured in both twinned and untwined volumes after different amounts of strain. The aim was to confirm whether twins are indeed intrinsically harder and to estimate what contribution this difference in hardness would make to the macroscopic flow stress. 


\section{Experimental}

\section{Material}

High-purity $\mathrm{CP}-\mathrm{Ti}$ and fine grain binary $\mathrm{Ti}-\mathrm{Al}$ alloys with $\mathrm{Al}$ concentrations from 0 to 13 at\% were produced at the Timet research facilities in Witton, UK. The CP-Ti and Ti-OAL aluminium materials were produced to have the same composition but heattreated to produce slightly different grain sizes. Buttons of $200 \mathrm{~g}$ were double-melted in a tungsten arc furnace under inert gas atmosphere followed by $\beta$-forging at $1100{ }^{\circ} \mathrm{C}$ and $\alpha$-cross-rolling in bar shape $(14 \times 14 \times 260 \mathrm{~mm})$ on a 2-high Robertson mill (WHA Robertson \& Co Ltd) at a temperature dictated by composition and subsequently recrystallized in the $\alpha$-phase region. The chemical composition of the alloys was determined by TIMET Savoie using a HORIBA Ultima2 for the metallic element analysis, a HORIBA EMIA $820 \mathrm{~V}$ combustion analyser for carbon analysis and a LECO EF-400 for oxygen and nitrogen analysis. The compositions are given in Table 1 . The starting CP-Ti material has a mean grain size of approximately $180 \mu \mathrm{m}$ with equiaxed morphology, as shown in Fig. 5a.

\section{Texture and orientation mapping}

EBSD orientation maps of the recrystallized material were obtained using step sizes of $1-3 \mu \mathrm{m}$ on a FEI Quanta 650 FEG-SEM with the AZtec software package from Oxford Instruments. Detailed orientation maps of deformed samples were always acquired with $1 \mu \mathrm{m}$ step size in order to capture twins. Macro-texture measurements were acquired with a $40 \mu \mathrm{m}$ step size on a CAMSCAN and a FEI Sirion FEG-SEM using the software Flamenco (Oxford Instruments) on areas larger than $16 \mathrm{~mm}^{2}$.

\section{Compression testing}

Cuboid $\left(6 \times 6 \times 9 \mathrm{~mm}^{3}\right)$ and cylindrical (either $6 \mathrm{~mm}$ diameter and $9 \mathrm{~mm}$ long or $8 \mathrm{~mm}$ diameter and $12 \mathrm{~mm}$ long) samples were machined with compression direction along the former rolling direction (RD) and normal direction (ND), as well as $45^{\circ}$ between both directions $\left(45^{\circ}\right)$, as shown in Fig. 1 . Note that there are two normal directions ND I and ND II, as the material was bar rolled; however, these directions were assumed to be identical, which was confirmed by the texture measurements. Uniaxial compression tests were conducted on an Instron 5569, an Instron 8562 and a MTS RT 100 to $3.7 \%$ and $8.7 \%$ plastic strain at room temperature and a strain rate of $1 \times 10^{-3} \mathrm{~s}^{-1}$.

\section{Nanoindentation}

Nanoindentation measurements can be significantly affected by surface deformation, and therefore, it is important that the surface is carefully prepared after sectioning. Although electropolishing would in principle guarantee a deformation-free surface, it was found that it produced pitting and hydride precipitation in samples studied here, especially after deformation. Therefore, after cross-sectioning, the samples were mechanically ground using increasingly fine resin-bonded diamond discs down to 550 grit size. After grinding, the samples were polished for about $5 \mathrm{~min}$ using diamond suspensions of decreasing size from 9 to $1 \mu \mathrm{m}$. Finally, to ensure the deformed surface layer was completely removed, a colloidal silica emulsion was used in a final long polishing step of $60 \mathrm{~min}$, with intermittent $10 \mathrm{~s}$ etching using Kroll's reagent $(94 \mathrm{ml}$ water $+4 \mathrm{ml}$ $\mathrm{HNO} 3+2 \mathrm{ml} \mathrm{HF}$ ) after $30 \mathrm{~min}$. Finally, the samples were cleaned in an ultrasonic bath to ensure the surfaces were clean. This procedure produced deformation-free surfaces in the recrystallized
Table 1 Chemical composition measured by TIMET Savoie and their grain size measured by the linear intercept method of the model alloys in this study

\begin{tabular}{lllllllc}
\hline Alloy & $\mathrm{Al}(\mathrm{wt} \%)$ & $\mathrm{Al}(\mathrm{at} \%)$ & $\mathrm{C}(\mathrm{ppm})$ & $\mathrm{N}(\mathrm{ppm})$ & $\mathrm{O}(\mathrm{ppm})$ & $\mathrm{Ti}$ & $\mathrm{GS}(\mu \mathrm{m})$ \\
\hline CP-Ti & 0.00 & 0.0 & 53 & 31 & 507 & Bal. & 180 \\
Ti-0Al & 0.00 & 0.0 & 55 & 13 & 474 & Bal. & 94 \\
Ti-3Al & 2.00 & 3.49 & 72 & 39 & 413 & Bal. & 82 \\
Ti-7Al & 3.90 & 6.67 & 50 & 23 & 592 & Bal. & 73 \\
Ti-10Al & 5.88 & 10.01 & 76 & 53 & 756 & Bal. & 71 \\
Ti-10Al & 5.88 & 9.99 & 76 & 53 & 567 & Bal. & 77 \\
Ti-13Al & 7.85 & 13.13 & 55 & 94 & 377 & Bal. & 78 \\
\hline
\end{tabular}




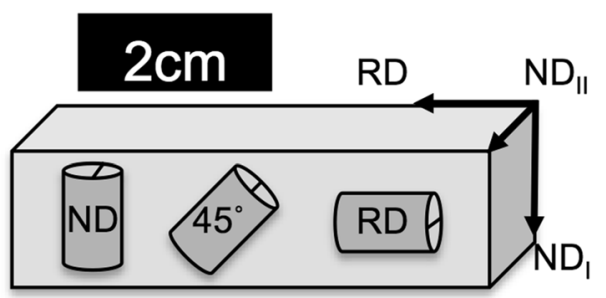

Figure 1 Macroscopic stress-strain curves CP-Ti with LD parallel to ND, RD and $45^{\circ}$.

materials, as confirmed by the quality of the EBSD patterns obtained. All samples were cross-sectioned and polished with the ND (normal direction) as surface normal, which also corresponds to the loading direction for the compressed samples.

Nanoindentation was carried out on samples mounted in Bakelite in a Nanoindenter XP from MTS equipped with a diamond Berkovich indenter. Indentations were made in arrays with more than $20 \mu \mathrm{m}$ spacing between indents and with a constant indentation rate of $0.05 \mathrm{~s}^{-1}$ to a depth of $500 \mathrm{~nm}$. The indenter was held for $10 \mathrm{~s}$ time at peak load for every measurement, and only measurement points that had a drift rate between sample and indenter head below $0.15 \mathrm{~nm} / \mathrm{s}$ were accepted. The nanohardness was calculated using the Oliver and Pharr method [16] as implemented in the Nanosuite software package. Stiffness measurements to monitor the modulus for each measurement were taken during the unloading to $50 \%$ of the maximum force. These were used to ensure the validity of the hardness values, which can be undermined by a loosely held sample, for example.

The indentation results were related to individual grain orientations measured by identifying the indentation locations on an EBSD orientation map. In order to minimise any effect of grain boundaries on the hardness value, indentations within $5 \mu \mathrm{m}$ to grain boundaries were discarded. In the case of twins, which were often narrower than $5 \mu \mathrm{m}$, only indents in the centre were considered.

\section{Results}

\section{Proof stress and stress-strain behaviour}

The $0.2 \%$ proof stress of the different alloys was determined by uniaxial compression testing. The stress-strain curves of the CP-Ti are shown in Fig. 2
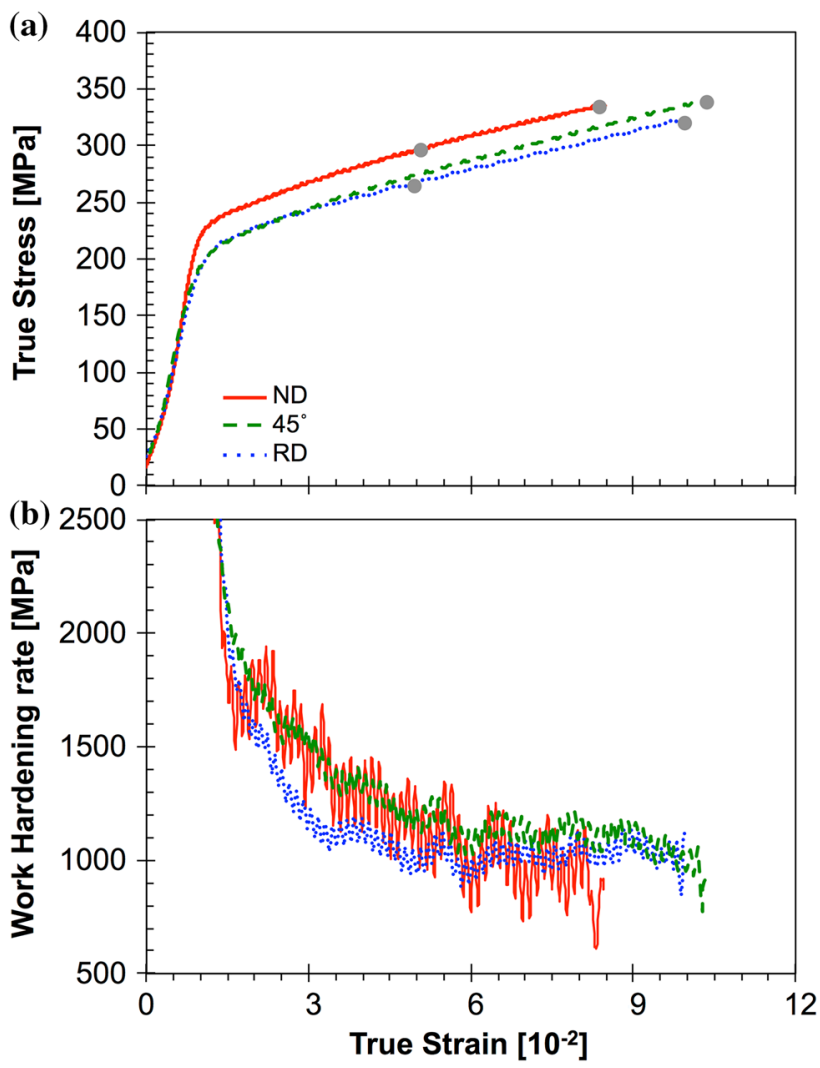

Figure 2 Macroscopic stress-strain curves CP-Ti with LD parallel to $\mathrm{ND}, \mathrm{RD}$ and $45^{\circ}$.

for loading along $\mathrm{RD}, \mathrm{ND}$ and $45^{\circ}$. It can be seen that the compressive proof stress is about $200 \mathrm{MPa}$ when loaded along RD and at $45^{\circ}$, while loading along ND gave a slightly higher proof stress of about $230 \mathrm{MPa}$. The work hardening rate is similar for all samples with the sample loaded along RD showing slightly lower values.

Figure 3 shows the yield stress, quantified using the $0.2 \%$ proof stress, of the $\mathrm{Ti}-\mathrm{Al}$ alloys for the three different loading directions. As for the CP-Ti sample, the proof stress is highest along ND and the lowest values along RD. These strength differences within one alloy can be explained by the texture, which is similar in all alloys (Fig. 4). A sample loaded along $\mathrm{RD}$ has more grains in a 'soft' orientation for which prismatic a slip is easy, whereas along ND there is a mixture of 'soft' and 'hard' grains, which results in a higher proof stress.

\section{Texture and microstructural evolution}

The texture changes after deformation are shown in Fig. 4 for the CP-Ti sample. Initially, the material has 

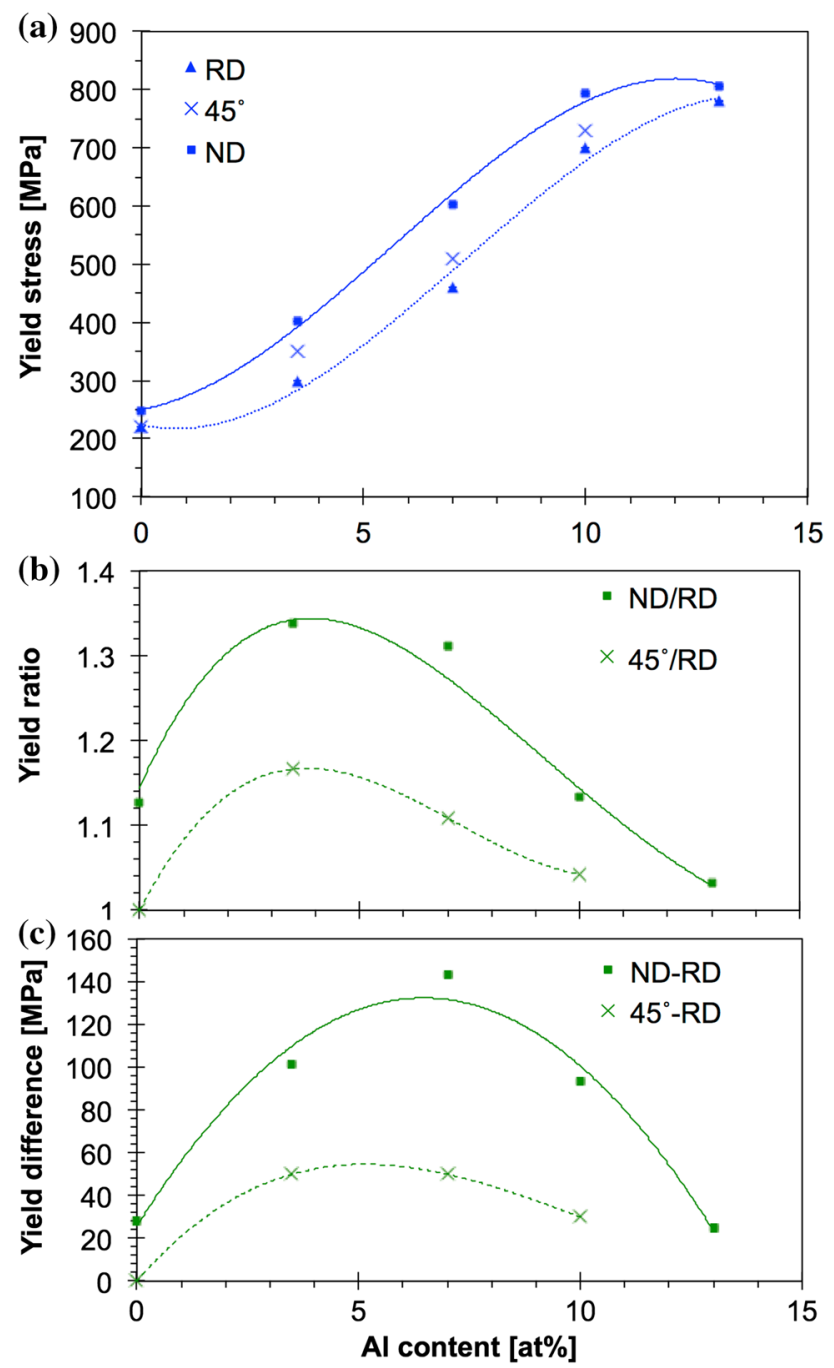

Figure 3 Yield anisotropy (quantified using 0.2\% proof stress), as function of the $\mathrm{Al}$ concentration: a yield point in $\mathrm{RD}, 45^{\circ}$ and $\mathrm{ND}, \mathbf{b}$ ratio of yield in ND to RD and $45^{\circ}$ to $\mathrm{RD}$.

a clockwork texture, with a preferred alignment of the basal poles aligns perpendicular to the rolling direction, without clear preference between $\mathrm{ND}_{\mathrm{I}}$ and $\mathrm{ND}_{\mathrm{II}}$. After $8 \%$ compression along ND, no significant texture change is observed although the intensities have reduced slightly, as shown in Fig. 4c. In contrast, $8 \%$ compression along RD increased noticeably the intensity of the basal pole in the loading direction, as shown in Fig. 4 d.

Figure $5 \mathrm{~b}$ shows an IPF map after $8 \%$ compression along ND. Misorientation profiles in grains that only underwent slip revealed gradients of up to $5^{\circ}$. In most grains, deformation twinning also occurred giving rise to misorientation angles of around $85^{\circ}, 35^{\circ}$ and $65^{\circ}$ for the twinning systems

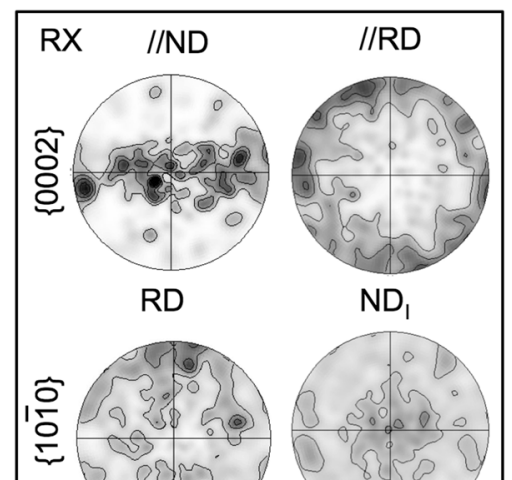

(a)

(b)
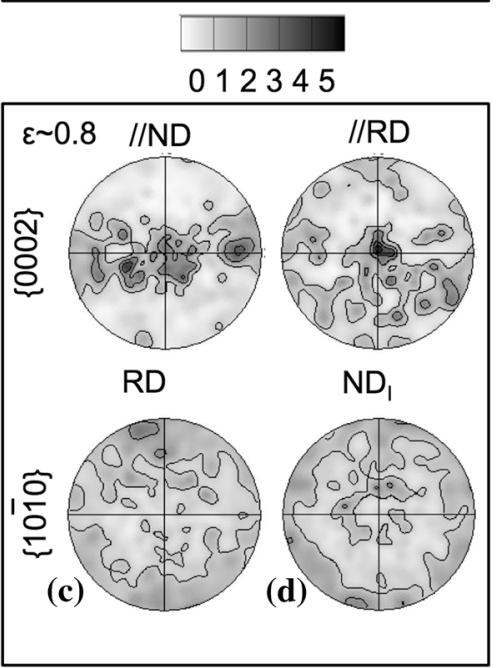

Figure 4 Basal and prismatic pole figures from macro-texture measurements after recrystallization (RX) along the ND (a) and (b) $\mathrm{RD}$ as well as at compressive strains of 0.09 along the ND (c) and $\mathrm{RD}(\mathbf{d})$.

$\{10 \overline{1} 2\} 10 \overline{1} 1,\{11 \overline{2} 1\} 1 \overline{1} 26$ and $\{11 \overline{2} 2\} 1 \overline{1} 23$, respectively. The $\{10 \overline{1} 2\} 10 \overline{1} 1$ tension twins are indicated by black twin boundaries and have a typical lenticular shape. Many grains exhibit multiple twin variants within one parent grain and consume considerable fractions of the parent grain, which makes them appropriate for nanoindentation of only the twinned volume. One example where the parent grain is nearly fully consumed is labelled as " $\mathrm{A}$ ", showing also secondary compression twins (marked with white boundaries). Hard grains, with their c-axis closer to the LD, exhibit lenticular or needle-shaped compression twins as primary twins, partly with secondary $\{10 \overline{1} 2\} 10 \overline{1} 1$ twins (example labelled as "B") and occasionally compression twins form within secondary tension twins. There are only a limited number of $\{11 \overline{2} 2\} 1 \overline{1} 23$ tension twins, which are 


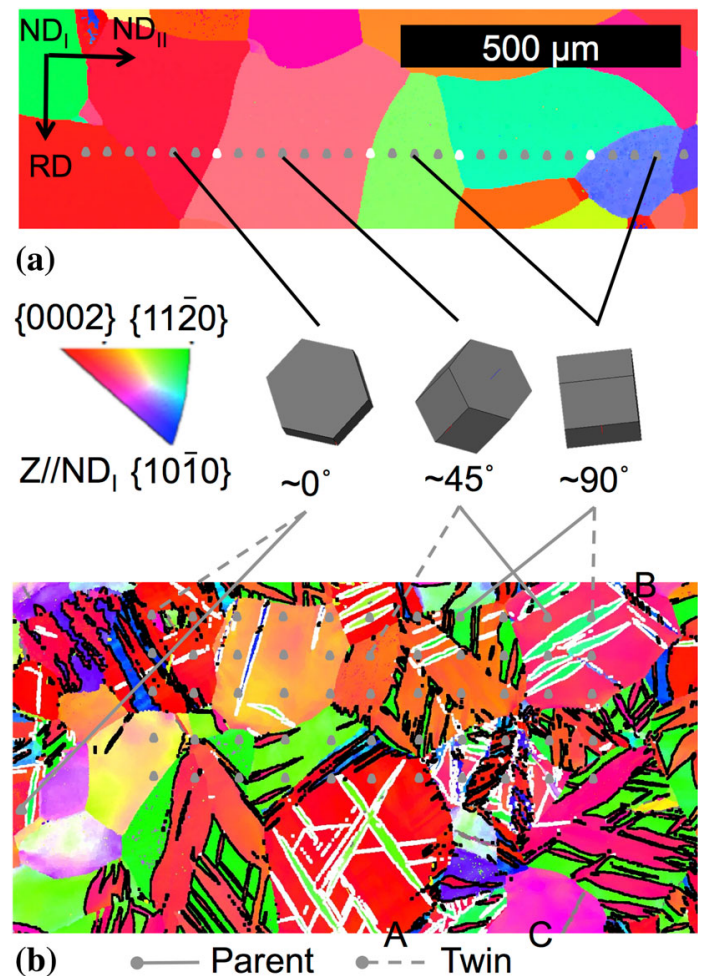

Figure 5 Schematic of methodology: EBSD maps in IPF-Z (ND) colouring from $\mathbf{a}$ big grained CP-Ti after RX treatment and $\mathbf{b}$ after $8 \%$ compression with indications for nanoindents (sizes not to scale) and the inclination angle between c-axis of the crystal and indentation direction (ID). For illustration full and dashed grey lines distinguish parent grains and twins, respectively.

always very thin $(<1 \mu \mathrm{m})$. They are indicated by grey twin boundaries, as in grain " $\mathrm{C}$ " in Fig. 5b.

Careful twin fraction analyses of orientation maps on more than $1 \mathrm{~mm}^{2}$ revealed that following $8 \%$ compression along $\mathrm{RD}$ there is a total twin area fraction of $31 \%$, with $28 \%\{10 \overline{1} 2\} 10 \overline{1} 1$ twins, $2.3 \%$ $\{1 \overline{1} 2\} 1 \overline{1} 23$ twins and less than $0.5 \%$ twins $\{10 \overline{1} 2\} 10 \overline{1} 1$. The large numbers of $\{10 \overline{1} 2\} 10 \overline{1} 1$ tension twins are responsible for the increase in basal pole intensity shown in Fig. 4d. Loading along ND results in dramatically reduced fraction of the $\{10 \overline{1} 2\} 10 \overline{1} 1$ twin, to an area fraction of $10 \%$, and slightly increased $\{11 \overline{2} 1\} 1 \overline{1} 26$ twin fraction, to about $10 \%$ area fraction. The frequency of $\{11 \overline{2} 1\} 1 \overline{1} 26$ twins also increases slightly to $1 \%$ area fraction. The combination of $\{10 \overline{1} 2\} 10 \overline{1} 1$ tension twinning and $\{11 \overline{2} 2\} 1 \overline{1} 23$ compression twinning has almost no effect on the texture, as can be seen in Fig. 4d). The twin volume fractions at different deformation stages are given in Table 2 .

\section{Nanohardness}

Nanoindentation was carried out on undeformed samples for all alloys and at two deformation levels for the CP-Ti sample. The undeformed CP-Ti and the $\mathrm{Ti}-\mathrm{OAl}$ materials were found to have the same hardness, and therefore their results are grouped together here. The arrays used for nanoindentation are shown schematically in Fig. 5. The orientation of the material under the indent was determined by overlapping the EBSD map with optical micrographs of the indented area. The indents are marked by white points in Fig. 5a were not considered because they lie within $5 \mu \mathrm{m}$ of a grain boundary. This condition makes the thin $\{11 \overline{2} 2\} 1 \overline{1} 23 \quad 35^{\circ}$ compression twins in the deformed samples challenging to measure without encountering boundary effects. However, reproducible results were possible by considering only measurements in the centre of the wider twins. The $\{11 \overline{2} 1\} 1 \overline{1} 26$ tension twins, however, were too thin to measure.

\section{Effect of Al content on nanohardness}

The nanohardness of the different alloys is plotted as a function of the angle between the c-axis and the indentation direction in Fig. 6. All samples showed a similar variation of hardness with angle, with indentations along the $c$ axis being significantly harder than perpendicular to it. For example, the $\mathrm{CP}$ $\mathrm{Ti}(\mathrm{Ti}-0 \mathrm{Al})$ sample displays a hardness of about 1.4 GPa along $a$ and about 2.5 GPa close to the $c$-axis. This corresponds to values of maximum forces per indent ranging from $10 \mathrm{mN}$ to $20 \mathrm{mN}$, respectively.

The plot shows that adding $\mathrm{Al}$ to $\mathrm{Ti}$ increases the nanohardness but has only a small effect on its variation with indentation direction. To show this
Table 2 Twin volume fraction in the CP-Ti material at the two different strain levels studied

\begin{tabular}{llll}
\hline Compressive strain (\%) & Tensile twins (\%) & Compressive twins (\%) & All twins (\%) \\
\hline 3 & 14 & 9 & 23 \\
8 & 39.5 & 13.1 & 52.6 \\
\hline
\end{tabular}




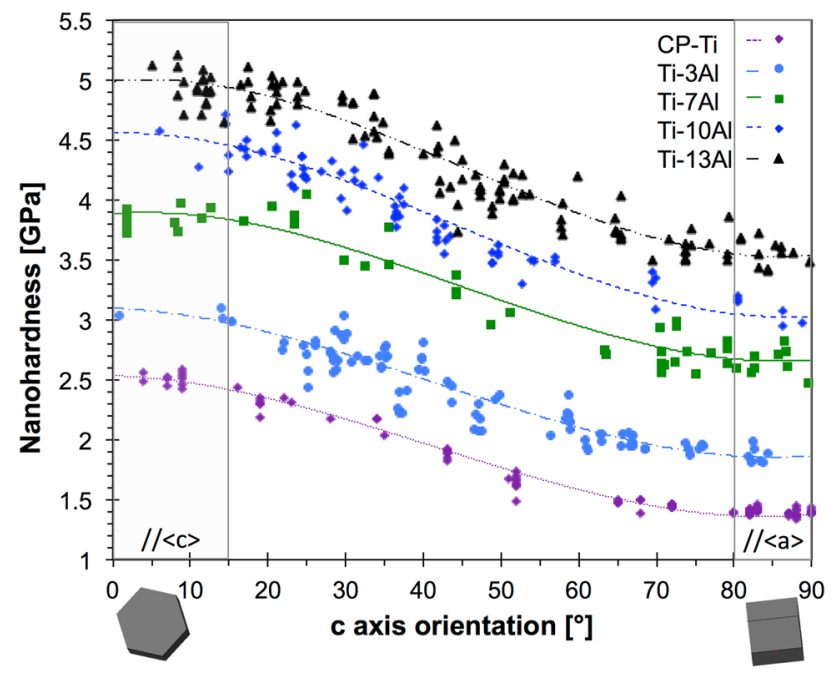

Figure 6 Nanohardness for several binary $\mathrm{Ti}-\mathrm{Al}$ alloys as function of the $c$ axis orientation. Angular ranges for averaging values in $a$ and $c$ directions are indicated.

more clearly, the nanohardness values were averaged for each alloy between $0^{\circ}$ and $15^{\circ}$ to represent the average hardness near the $c$ direction and between $80^{\circ}$ and $90^{\circ}$ for the $a$ direction, as indicated in Fig. 6. Figure $7 \mathrm{a}$ plots these averages as function of $\mathrm{Al}$ concentration with the error bars reflecting the range of data in the described angular regimes for $a$ and $c$, rather than the standard deviation. Values in the soft a direction increase almost linearly from approximately 1.4 GPa in Ti-0Al to 3.5 GPa in Ti-13Al, while the nanohardness in the hard $c$ direction increases from 2.5 to $5 \mathrm{GPa}$, although the effect is slightly smaller from 0 to 3 at\% and from 10 to 13 at $\%$.

The hardness ratio between $a$ and $c$ indentations decreases marginally with $\mathrm{Al}$ addition as shown in Fig. $7 \mathrm{~b}$. The difference between hardness along $a$ and $c$ increases only slightly with $\mathrm{Al}$ content and within the measurement scatter (see Fig. 7c). Despite the strong effect of alloying on hardness, a grain close to the soft $a$ direction in an alloy of high Al concentration can be softer than a hard grain of an alloy containing less $\mathrm{Al}$. For instance, Ti-13Al exhibits a lower nanohardness along the $a$ direction (3.5 GPa) than Ti$7 \mathrm{Al}$ along the $c$ direction (3.8 GPa).

\section{Effect of deformation on nanohardness}

To investigate the effect of deformation on the nanohardness, the CP-Ti material was tested at two levels of strain: $4 \%$ along RD and ND and after approximately $8-9 \%$ (8.7 in RD, $7.7 \%$ in ND and $9.2 \%$
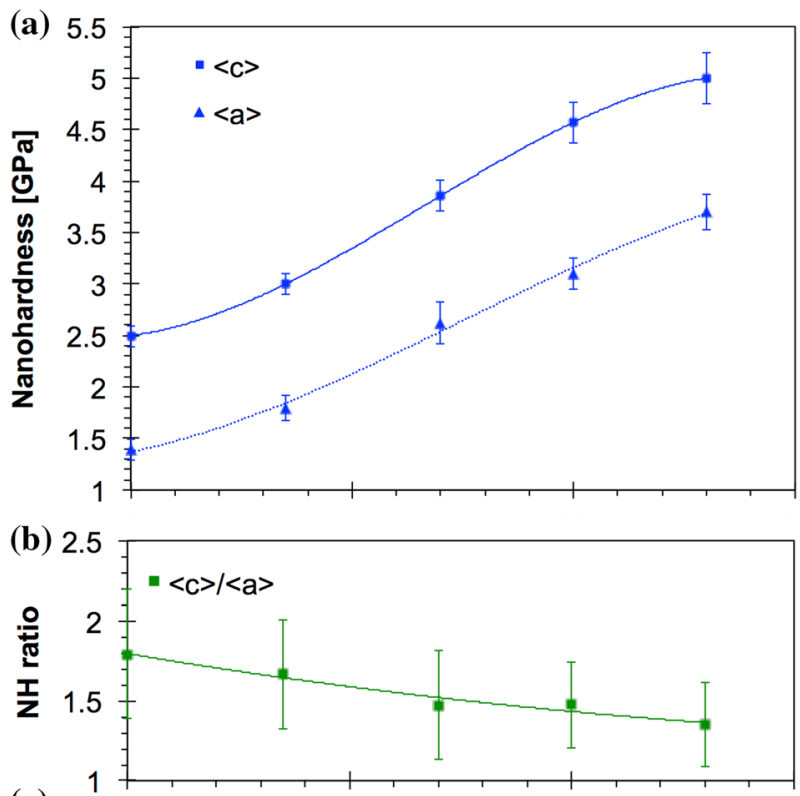

(c)

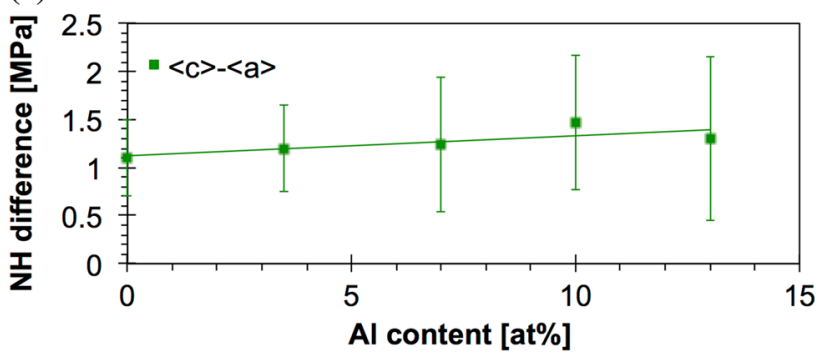

Figure 7 Nanohardness for hard (along) and soft (along) indentations. a Absolute values. b Hardness rations. c Hardness difference.

at $45^{\circ}$ ). This corresponds to an increase in the flow stress due to work hardening of approximately $60 \mathrm{MPa}$ at $4 \%$ and about $120 \mathrm{MPa}$ at the higher strain level, as indicated by the grey circles in Fig. 2a. The results of the measurements on the strained samples are plotted alongside those in the recrystallized material in Fig. 8, showing that the strained material is slightly harder than the undeformed material. However, the mean increase in hardness with straining is small and less than the variation in hardness with orientation. There is also a noticeable increase in measurement scatter with straining.

Despite the scatter, the data reward careful study. The plots in Fig. 8 compare the hardness of the undeformed material with that of deformed untwined grains (Fig. 8a) and with that of twins (Fig. 8a). Because the scatter makes it difficult to quantify the differences, linear regression lines were fitted to the low-angle region (between $0^{\circ}$ and $65^{\circ}$ ) 
(a)

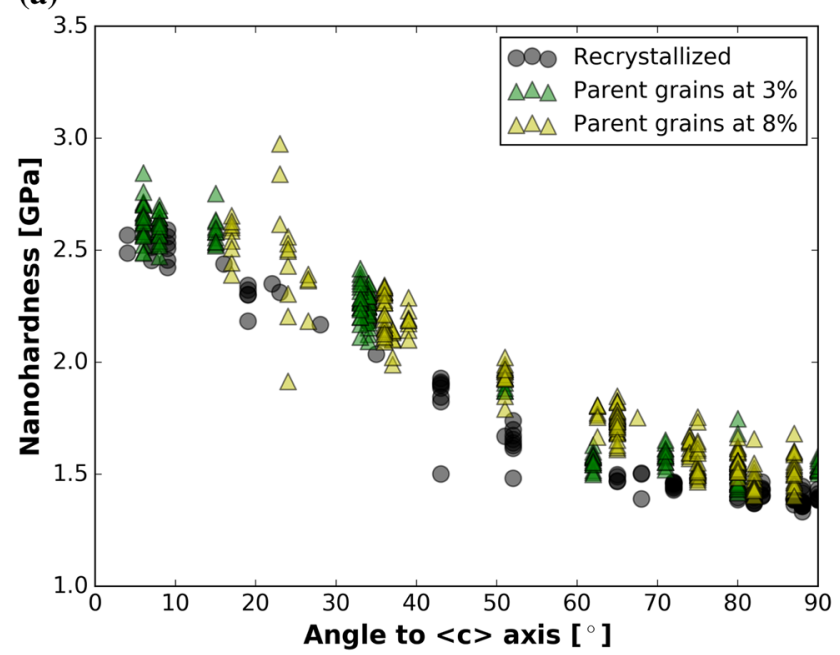

(c)

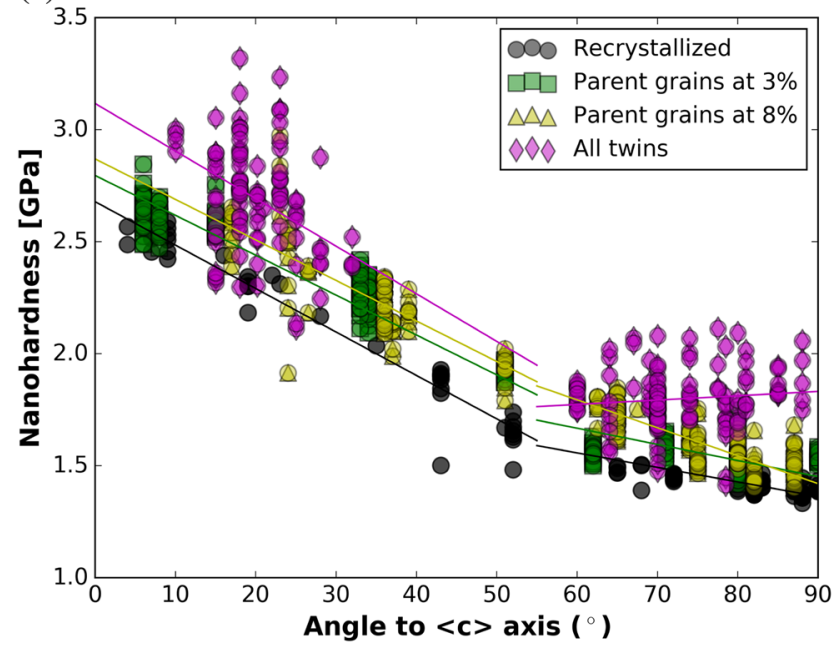

Figure 8 Comparison of nanohardness in recrystallized and deformed CP-Ti. Hardness is plotted against the inclination of the $c$ axis with respect to indenting direction: a comparison with

and another to the high-angle region (between $50^{\circ}$ and $90^{\circ}$ ) as shown in Fig. 8c. The mean distance between these lines was then used to estimate the hardness increase with deformation, which is given in Fig. 8d). If one considers the non-twinned regions, the increase in hardness at $4 \%$ strain is only about 0.1-0.15 GPa and increases only by 0.05 to $0.07 \mathrm{GPa}$ with further straining to $8 \%$. On the other hand, most indents within twins show a higher hardness than indents in non-twinned grains of comparable orientation. The twins at higher angles can have hardness values $0.5 \mathrm{GPa}$ higher that the deformed grains and $0.8 \mathrm{GPa}$ higher than undeformed grains, although the (b)

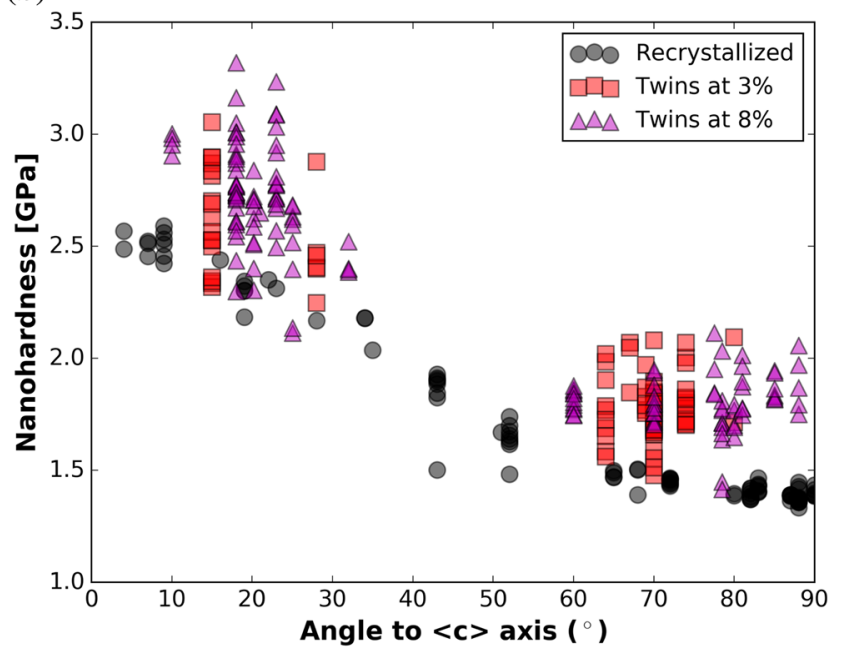

(d)

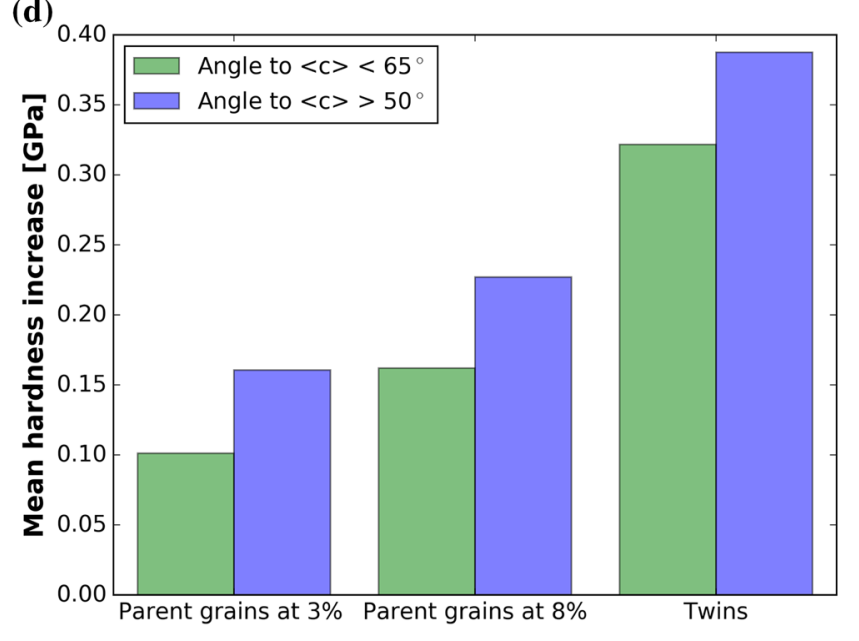

deformed parent grains only $\mathbf{b}$ comparison with twinned grains only $\mathbf{c}$ all data with fitted regression lines $\mathbf{d}$ summary of hardness increases for high and low inclination angles.

difference between average hardness is 0.25 and 0.4 $\mathrm{GPa}$, respectively. The twins at lower angles also show higher hardness values than the deformed nontwinned grains, although the difference is slightly smaller than at higher angles. The measurements within the twins also show much more scatter. However, there is no significant difference in the hardness values of the twins at $4 \%$ strain and the twins at $8 \%$, as can be seen clearly in Fig. $8 \mathrm{~b}$. 


\section{Discussion}

\section{Orientation dependence of nanohardness}

The absolute values of hardness obtained show good agreement with previous measurements on $\mathrm{CP}-\mathrm{Ti}$ $[7,15,17,18]$ and Al-strengthened alpha-Ti [17, 19]. The work by Britton et al. shows abnormally high absolute values of hardness in CP-Ti than observed in the present study, although the difference in hardness between the highest and lowest value is similar at $1 \mathrm{GPa}$ [20].

Interpreting the variation in hardness with orientation in terms of relative slip activity is not straightforward since several interacting slip systems are activated during indentation. Work by Kwon et al. [19] on $\mathrm{Ti}-7 \mathrm{wt} \% \mathrm{Al}(\sim \mathrm{Ti}-11.5$ at\% $\mathrm{Al})$ with a spherical nanoindenter and Viswanathan et al. with a Berkovich indenter on Ti6Al4 V [17] showed that indentation along the soft prismatic directions can in principle activate $a$ slip on basal, prismatic and pyramidal planes but also give rise to pyramidal $c+a$ slip and twinning. They also detected evidence of activity on all slip systems for hard indentations (within $5^{\circ}$ of the $c$ axis) although prismatic slip activity was much lower than for the softer orientations. Viswanathan et al. also found $c$-axis and $c+a$ type dislocations during indentation of Ti6Al4 V $15^{\circ}$ from the $c$ axis with a Berkovich indenter. Crystal plasticity finite element simulations of indentation depth and pile-up also suggest that regardless of the grain orientation all slip systems are activated to some extent but that prismatic slip dominates in the soft orientations [18]. This explains the strong effect of indentation direction on the hardness measured.

\section{Nanohardness and proof stress of Ti-Al alloys}

Increasing the $\mathrm{Al}$ content increases both the flow stress and the hardness in very similar ways, with smaller increases between 0 and 3 and between 10 and 13 at $\%$ than those at intermediate $\mathrm{Al}$ contents. Thus, the relationship between proof stress and hardness is essentially linear, as can be seen in Fig. 9. The slope depends slightly on which loading directions are used for the comparison: For indentation along $c$ and RD compression, the slope is 3.8, whereas for indentation along $a$ and ND compression, it is about 4.3. In other words, a $100 \mathrm{MPa}$ increment in

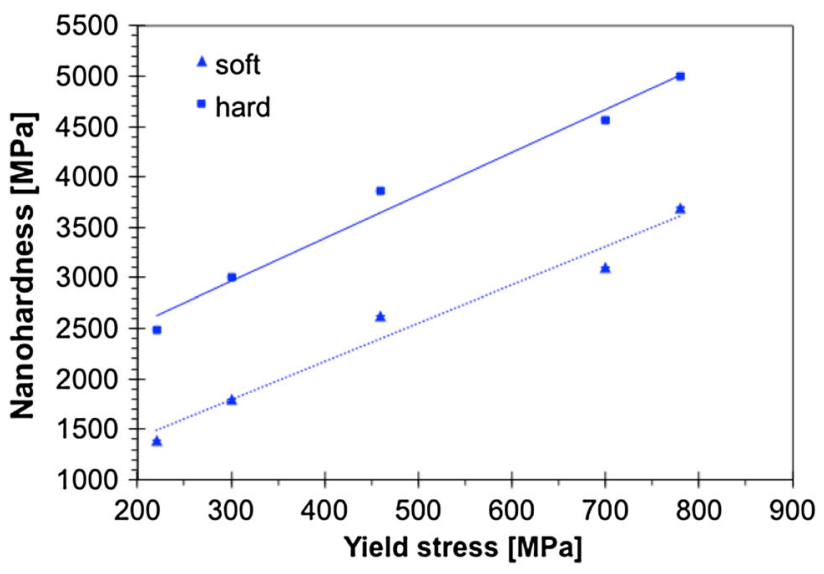

Figure 9 Plot of nanohardness against proof (yield) stress for the different alloys tested, where hard indicates grains aligned between 0 and $15^{\circ}$ to the $\langle c\rangle$ axis and "soft" indicates indents at 80-90 .

flow stress corresponds to a change in hardness of about 0.4 GPa.

The nanoindentation results in Fig. $7 \mathrm{~b}$ show that the hardness becomes more isotropic with $\mathrm{Al}$ addition. It is tempting to explain this decrease in anisotropy to changes in the slip anisotropy. Small changes in anisotropy with $\mathrm{Al}$ content have also been reported by Williams et al. [21], where $\mathrm{Al}$ addition makes basal slip relatively easier compared to prism slip but $c+a$ slip relatively more difficult than $a$ slip. However, since all slip systems are active for all loading directions, this decrease in anisotropy could simply be due to the increase in strength caused by $\mathrm{Al}$ addition. Since the increase in strength from $\mathrm{Al}$ is an additional term, increasing the slip resistance of all slip systems by the same amount will have the effect of decreasing the anisotropy as defined by the ratio of slip resistances. This is supported by the fact that the ratio of yield strength for the $45^{\circ}$ case to that of the $\mathrm{RD}$ case decreases from 3 at $\% \mathrm{Al}$ with increasing $\mathrm{Al}$ addition, as would be expected from the overall higher strength levels with similar differences between both directions.

It is then surprising that the proof stress of the Ti$0 \mathrm{Al}$ sample is the most isotropic. The proof stress when compressed in RD and $45^{\circ}$ is identical and the proof stress along ND is only slightly higher than that along RD even though the texture is not different from that in the binary alloys and the nanohardness values show identical variation with indentation direction. The reason for this is likely to be the very low initial slip resistance in the Ti-0Al material and a 
significant contribution of initial hardening to the proof stress values measured. As discussed by Hutchinson and Barnett [22], if the initial slip resistance is very low, then a small absolute increment in hardening with straining has a disproportionate effect on the slip resistance. It is plausible that the proof stress measurement in this material is affected by a high rate of initial work hardening caused by slip and that since this effect would be strongest for $\mathrm{RD}$ and $45^{\circ}$, it acts to reduce the measured anisotropy.

\section{Nanohardness of the deformed material}

The hardening behaviour observed during compression testing to just under $10 \%$ is almost independent of direction (Fig. 2b). At 3\% the flow stress has increased by $60 \mathrm{MPa}$ and at $9 \%$ by about $120 \mathrm{MPa}$ for all loading directions. This change in flow stress is accompanied by a change in hardness in non-twinned grains, which is, however, only detectable for the first strain increment. This suggests that the increase in flow stress observed during compression testing is only partly caused by a change in the local flow resistance, and that this hardening occurs in the very early stages of deformation. Using the relationship between nanohardness and flow stress developed for the Alcontaining alloys, the $0.1 \mathrm{GPa}$ change in hardness observed translates to a $30 \mathrm{MPa}$ change in flow stress, about half the change in flow stress at $4 \%$ and less than a quarter of the flow stress measured at $\sim 8 \%$.

The twinned regions show a higher hardness than both the non-deformed grains and the deformed, nontwinned regions. However, this hardness increase is smaller than the change in hardness with orientation, in contradiction with the results by Salem et al. [7]. Nevertheless, it is clear that twins are harder than nondeformed grains or deformed parent grains of comparable orientation. The mean difference in hardness is about $0.4 \mathrm{GPa}$, which corresponds to a change in flow stress of $100 \mathrm{MPa}$. Although this increase in hardness is higher than that in the non-twinned regions, it is still less than would be expected from the change in flow stress by work hardening (120 MPa).

Since the indentation direction is always parallel to the loading direction during compression testing, the results from tension twins can be easily separated from those of compression twins. Tensile twinning causes a rotation of the $c$ axis towards the LD, whereas compression twinning causes the $c$ axis to rotate away from the LD. Therefore, all twins at low indentation angles $\left(0-30^{\circ}\right)$ will be tensile twins, whereas the twins at high angles will be compression twins.

The scatter in the nanoindentation measurements is higher in the twins than in the deformed grains, and the hardness in the twins after $4 \%$ strain is identical to that after 9\% strain. Furthermore, the increase in hardness is higher for the compression twins than the tensile twins. Three possible hardening mechanisms will be considered that could explain the higher twin hardness: change in slip length (HallPetch effect), the Basinski mechanism and residual twinning stresses.

The slip length mechanism is difficult to test since the origin of the Hall-Petch effect is not fully understood. However, it is difficult to see how slip length effects could be detected by nanoindentation, given the small volume of material deformed in the test. So, although changes in slip length due to twinning might contribute to changes in flow stress, it cannot explain the higher hardness measured within the twins.

The Basinski mechanism, by which dislocations are made sessile by twinning, could offer an explanation for the increased hardness measure. In their paper, Salem et al. [7] propose that the higher hardness of the twins is experimental evidence of this mechanism. However, it appears that, in their work, measurements made on twins at low angles were compared to parent grains at high angles and therefore the difference in hardness measured is mostly a result of orientation. If the increase in hardness arises from the generation of an array of sessile dislocations during twinning, as predicted by the Basinski mechanism, then this effect should be more pronounced for grains containing more mobile dislocations before twinning. Since, for uniaxial compression, compression twins occur primarily in hard grains where twinning precedes slip, they should show almost no increase in hardness, whereas tensile twins, which appear in grains ideally aligned for easy prismatic slip, should show a large increase in hardness. Furthermore, one would expect that the hardness in the twins would be higher at higher strains, since they will be more likely to contain a larger number of dislocations. However, the results clearly show that there is no difference in the hardness at different strain levels and that the difference in hardness is larger for the compression twins than 
for the tensile twins. Therefore, these new results do not support the Basinski or other twinning-dislocation interaction mechanism.

Significant local residual stresses are generated during twinning, as the twinning shear introduces a misfit strain in the parent grain. These stresses are the reason why twins are lenticular. In a recent numerical study by Arul Kumar et al. [23], it was shown that the twin is constrained by parent grain, which acts to reduce the shear stress in the twinning plane inside the twin. The magnitude of the stresses in the twins depends on the ability of the parent grain and of the neighbouring grains to accommodate the misfit strain by plastic deformation but is mostly determined by the twinning transformation. There is also direct experimental evidence for this local stress reduction (sometimes called reversal) [24]. Such stresses will of course also affect the resolved shear stresses for slip inside the twin during deformation. In the unloaded condition and at a polished surface, these stresses will remain as residual in-plane compressive stresses, which are well known to cause an increase in the measured nanohardness [25]. If the stresses are mostly determined by the transformation shear, then they should be higher in the compression twins (shear strain of 0.225) than in the tension twins (shear strain of 0.167). This agrees with the results in Fig. 8, where it is clear that the increase in hardness in the compression twins is higher than that in the tension twins. The residual stress argument also explains why the twin hardness is the same after 3\% and $8 \%$ straining, since the stresses are dominated by the twinning shear strain, which does no change with a few $\%$ of deformation. It also helps explain why the scatter in the twins is higher than that in parent grains, since the stresses within a twin vary significantly with position [23], and hence will cause the measured hardness to vary with indentation location.

In summary, these measurements confirm that the nanohardness of the twins is higher than that in the parent grains. However, this difference is small and much smaller than the total variation with orientation. Furthermore, these results suggest that the higher hardness of the twins is caused by residual stresses and not a Basinski-type mechanism.

\section{Implications for work hardening behaviour}

In light of these new results, the different explanations for the effects of twinning on the work hardening are now reconsidered. Our measurements imply that the changes in local slip resistance, as measured by nanoindentation, can only explain a small part of the observed hardening. Contrary to previous work, these nanoindentation measurements provide no evidence for work hardening induced by twinning-dislocation interactions.

The results do highlight, however, the strong plastic anisotropy of $\mathrm{Ti}$, which implies that, especially at small strains (and low applied stresses), deformation by slip will be very heterogeneous, occurring predominantly on soft oriented grains, well oriented for prismatic and maybe basal slip. The hard orientations raise the mean flow stress due to their low Schmid factor for prismatic slip and, in addition, the misfit between hard and soft grains will give rise to back stresses, which will contribute to work hardening. These stresses are also probably at least partly responsible for the strong Bauschinger effect observed in CP-Ti [4].

However, twinning can increase the fraction of hard grains potentially be a potent hardening mechanism. However, only tensile twinning should promote hardening in this way, whereas compression twinning should give rise to texture softening. Therefore, reorientation caused by tensile twinning can explain the peak in hardening rate observed for compression along soft directions, like RD but not the strong hardening observed when loading along hard directions, such as ND, where compression twinning would be expected to counteract some of the texture hardening caused by tension twinning. If the texture is not very strong, as in the case of the material studied here, primary tensile twinning will also take place and primary compression twins readily form secondary tension twins. But even for stronger textures and at low temperatures, hardening is observed. For compression loading, the evidence is that both tensile twinning and compressive twinning contribute to the work hardening of the material [5]. This implies that reorientation softening from compression twinning is less effective than reorientation hardening from tensile twinning.

To try to explain this difference, the differences between the two twinning modes must be considered. The first is the reorientation angle: $\{10 \overline{1} 2\} 10 \overline{1} 1$ tensile twinning reorients the lattice by about $85^{\circ}$, whereas the reorientation from $\{11 \overline{2} 2\} 1 \overline{1} 23$ compressive twinning is only $65^{\circ}$. Thus, the reorientation 
effect will be stronger for tensile twins, especially in $\mathrm{Ti}$, where prismatic slip is dominant. The other difference is that, in compression loading, tensile twinning usually occurs in grains well oriented for prismatic slip, whereas compression twins appear in hard grains, where prismatic slip is difficult. This means that the potential for relieving the twinninginduced misfit stresses by slip is easier in the tensile case. Since these stresses act to hinder twin growth [23], this explains why tensile twins grow to cover entire grains, while compressive twins usually remain thin.

This is also likely to be the reason for the work hardening observed for compression along hard directions like ND and at low temperatures. Since the twinning stresses in compressive twins cannot be easily relieved by slip, twins cannot grow and instead multiple variants are generated in each grain. This not only becomes increasingly difficult with deformation, but because the twinning length of later generation twins is reduced they can contribute less to the macroscopic strain, giving rise to work hardening.

The presence of these thin compression twins and many twin intersections makes the idea that reduced slip length could contribute to hardening via a HallPetch-type effect very compelling. However, the parent grains are hard and do not deform much, if at all, before twinning and therefore the creation of new boundaries should not change the macroscopic flow stress even if the slip length is reduced. At small strain, the size effect is probably in limiting twin length, not slip length. If there is an effect on slip resistance at higher strains, it is that of local twinning stresses which will act to lower the shear stress in the reoriented twinned volume, making it appear harder than its orientation alone would suggest. Such stresses would be strongly directional which is consistent with the finding that the slip length effect can be better represented by change in latent hardening than an isotropic size effect in crystal plasticity models [11].

Therefore, twinning stresses offer a simpler explanation than that put forward by El Kadiri and Oppedal [8] based on dislocation transmutation. The difficulty in relaxing the twinning stress in the compressive case explains why reorientation softening by compressive twinning is not as significant as expected. So if compressive twinning is dominant, the material will work harden as twinning becomes more difficult with the expected softening induced by reorientation thwarted by the generation of twinninginduced back stresses, which also increase the measured local hardness.

This leaves unexplained results from the temperature reversal experiments by Chichili et al. on Ti [6] and Kaschner et al. on $\mathrm{Zr}$ [12] where samples deformed at $77 \mathrm{~K}$ have a lower yield stress on reloading than the samples deformed at room temperature, suggesting a strong softening effect induced by reorientation during compression twinning. In these experiments, low deformation temperatures were used to promote twinning at the expense of slip. However, the researchers have not considered the strong thermal anisotropy of these materials and the associated thermal residual stress relaxation that will occur on reheating to room temperature. Ti has been shown to have an unusually large thermal anisotropy at liquid nitrogen temperatures, partly as a result of an anomalous thermal expansion behaviour, where the thermal expansion along the $c$ axis becomes negative below $170 \mathrm{~K}$, while along $\langle a\rangle$ it remains constant at about $9 \times 10^{-6} \mathrm{~K}^{-1}$ [26]. This means than, on reheating, the stresses induced by compressive twinning will be relaxed by the differential thermal expansion, hence reducing the back stresses on the reoriented twin volumes and decreasing the overall flow stress. This would also explain the need to add "recovery" on reheating when modelling this behaviour [27]. On reloading at RT, further twinning will induce new stresses, which explains why the original flow stress and work hardening rate are eventually recovered. Therefore, twinning stresses not only explain the slightly higher nanohardness of twins, but also explain the unusual temperature reversal experiments, further strengthening the argument that they are an important part of understanding how twinning affects work hardening.

\section{Conclusions}

Careful nanoindentation measurements have shown that although the hardness of CP-Ti does increase slightly with deformation, the change in hardness in the untwinned grains is insufficient to explain the changes in flow stress. Twinned volumes are slightly harder than untwined regions. However, the results suggest, but do not prove, that this increase is caused by the presence of residual twinning stresses and not due to a Basinski-type mechanism. 
During compressive straining along soft directions, like $\mathrm{RD}$, the main contribution of twinning to hardening is by reorientation-induced hardening through tensile twinning. Along hard directions, where the contribution of tensile twinning is small, it is suggested that work hardening is caused by the saturation of twinning and strong latent hardening in the twinned volumes, both of which could be a direct consequence of the difficulty in relaxing the twinning-induced stresses. These can be relieved by exploiting the strong thermal anisotropy of these materials, as demonstrated by temperature reversal experiments.

Most of the attempts to explain the work hardening in $\mathrm{Ti}$ have focused on twinning-dislocation interaction mechanisms and changes in slip length caused by twinning. In contrast, the development of internal stresses at the microstructural scale has mostly been ignored. These new results suggest that internal stresses could play a role in the work hardening behaviour of $\mathrm{Ti}$, both through the development of back stresses between hard and soft oriented grains and twins, and through the twinning process itself. Since these stresses can be accommodated by plastic deformation in the twin, parent grain and even neighbouring grains, there will be a strong effect of slip activity on twinning. This is of great relevance for Al-containing Ti alloys, where slip occurs at much higher stresses [28].

\section{Acknowledgements}

The authors thank Andrew Forrest for assistance during the nanoindentation and the EPSRC for funding this project through the programme grants LATEST2 (Grant code EP/H020047/1) and LightForm (EP/R001715/1). All data presented here are available on request.

\section{Funding}

This study was funded by EPSRC (Grant codes EP/ H020047/1 and EP/R001715/1).

\section{Compliance with ethical standards}

Conflict of interest Dr Matthew Thomas is an employee of TIMET who supplied the material used in this study. Dr João Quinta da Fonseca is an editor of the Journal of Materials science. No other conflicts of interest exist.

Open Access This article is distributed under the terms of the Creative Commons Attribution 4.0 International License (http://creativecommons.org/ licenses/by/4.0/), which permits unrestricted use, distribution, and reproduction in any medium, provided you give appropriate credit to the original author(s) and the source, provide a link to the Creative Commons license, and indicate if changes were made.

\section{References}

[1] Partridge PG (1967) The crystallography and deformation modes of hexagonal close-packed metals. Metall Rev 12:169-194. https://doi.org/10.1179/mtlr.1967.12.1.169

[2] Hama T, Nagao H, Kobuki A, Fujimoto H, Takuda H (2015) Work-hardening and twinning behaviors in a commercially pure titanium sheet under various loading paths. Mater Sci Eng A 620:390-398. https://doi.org/10.1016/j.msea.2014.10. 024

[3] Salem AA, Kalidindi SR, Doherty RD (2003) Strain hardening of titanium: role of deformation twinning. Acta Mater 51:4225-4237. https://doi.org/10.1016/S1359-6454(03)002 $39-8$

[4] Nixon ME, Cazacu O, Lebensohn RA (2010) Anisotropic response of high-purity $\alpha$-titanium: experimental characterization and constitutive modeling. Int J Plast 26:516-532. h ttps://doi.org/10.1016/j.ijplas.2009.08.007

[5] Battaini M, Pereloma EV, Davies CHJ (2007) Orientation effect on mechanical properties of commercially pure titanium at room temperature. Metall Mat Trans A 38:276-285. https://doi.org/10.1007/s11661-006-9040-2

[6] Chichili DR, Ramesh KT, Hemker KJ (1998) The highstrain-rate response of alpha-titanium: experiments, deformation mechanisms and modeling. Acta Mater 46:1025-1043. https://doi.org/10.1016/S1359-6454(97)002 $87-5$

[7] Salem AA, Kalidindi SR, Doherty RD, Semiatin SL (2006) Strain hardening due to deformation twinning in $\alpha$-titanium: mechanisms. Metall Mat Trans A. 37:259-268. https://doi. org/10.1007/s11661-006-0171-2

[8] El Kadiri H, Oppedal AL (2010) A crystal plasticity theory for latent hardening by glide twinning through dislocation transmutation and twin accommodation effects. J Mech Phys Solids 58:613-624. https://doi.org/10.1016/j.jmps.2009.12. 004 
[9] Salem AA, Kalidindi SR, Semiatin SL (2005) Strain hardening due to deformation twinning in $\alpha$-titanium: constitutive relations and crystal-plasticity modeling. Acta Mater 53:3495-3502. https://doi.org/10.1016/j.actamat.2005.04. 014

[10] Proust G, Tomé CN, Kaschner GC (2007) Modeling texture, twinning and hardening evolution during deformation of hexagonal materials. Acta Mater 55:2137-2148. https://doi. org/10.1016/j.actamat.2006.11.017

[11] Oppedal AL, El Kadiri H, Tomé CN, Kaschner GC, Vogel SC, Baird JC et al (2012) Effect of dislocation transmutation on modeling hardening mechanisms by twinning in magnesium. Int J Plast 30-31:41-61. https://doi.org/10.1016/j.ijpla s.2011.09.002

[12] Kaschner GC, Tomé CN, McCabe RJ, Misra A, Vogel SC, Brown DW (2007) Exploring the dislocation/twin interactions in zirconium. Mater Sci Eng A 463:122-127. https://d oi.org/10.1016/j.msea.2006.09.115

[13] Capolungo L, Marshall PE, McCabe RJ, Beyerlein IJ, Tome $\mathrm{CN}$ (2009) Nucleation and growth of twins in $\mathrm{Zr}$ : a statistical study. Acta Mater 57:6047-6056. https://doi.org/10.1016/j. actamat.2009.08.030

[14] Morrow BM, Cerreta EK, McCabe RJ, Tomé CN (2015) Transmission electron microscope in situ straining technique to directly observe defects and interfaces during deformation in magnesium. JOM 67:1-8. https://doi.org/10.1007/s11837015-1432-6

[15] Mante FK, Baran GR, Lucas B (1999) Nanoindentation studies of titanium single crystals. Biomaterials 20:1051-1055. https://doi.org/10.1016/S0142-9612(98)002 $57-9$

[16.] Oliver WC, Pharr GM (2004) Measurement of hardness and elastic modulus by instrumented indentation: advances in understanding and refinements to methodology. J Mater Res 19:3-20. https://doi.org/10.1557/jmr.2004.19.1.3

[17] Viswanathan GB, Lee E, Maher DM, Banerjee S, Fraser HL (2005) Direct observations and analyses of dislocation substructures in the $\alpha$ phase of an $\alpha / \beta$ Ti-alloy formed by nanoindentation. Acta Mater 53:5101-5115. https://doi.org/ 10.1016/j.actamat.2005.07.030

[18] Zambaldi C, Yang Y, Bieler TR, Raabe D (2012) Orientation informed nanoindentation of $\alpha$-titanium: indentation pileup in hexagonal metals deforming by prismatic slip. J Mater Res 27:356-367. https://doi.org/10.1557/jmr.2011.334

[19] Kwon J, Brandes MC, Phani PS, Pilchak AP, Gao YF, George EP, Pharr GM, Mills MJ (2013) Characterization of deformation anisotropies in an $\alpha$-Ti alloy by nanoindentation and electron microscopy. Acta Mater 61:4743-4756. http s://doi.org/10.1016/j.actamat.2013.05.005

[20] Britton TB, Liang H, Dunne FPE, Wilkinson AJ (2010) The effect of crystal orientation on the indentation response of commercially pure titanium: experiments and simulations. Proc R Soc A 466:695-719. https://doi.org/10.1098/rspa.20 09.0455

[21] Williams JC, Baggerly RG, Paton NE (2002) Deformation behavior of HCP Ti-Al alloy single crystals. Metall Mat Trans A 33:837-850. https://doi.org/10.1007/s11661-002-0 153-y

[22] Hutchinson WB, Barnett MR (2010) Effective values of critical resolved shear stress for slip in polycrystalline magnesium and other hcp metals. Scripta Mater 63:737-740. https://doi.org/10.1016/j.scriptamat.2010.05.047

[23] Kumar MA, Kanjarla AK, Niezgoda SR, Lebensohn RA, Tomé CN (2015) Numerical study of the stress state of a deformation twin in magnesium. Acta Mater 84:349-358. h ttps://doi.org/10.1016/j.actamat.2014.10.048

[24] Aydıner C, Bernier J, Clausen B, Lienert U, Tomé C, Brown D (2009) Evolution of stress in individual grains and twins in a magnesium alloy aggregate. Phys Rev B 80:024113. h ttps://doi.org/10.1103/PhysRevB.80.024113

[25] Tsui TY, Oliver WC, Pharr GM (1996) Influences of stress on the measurement of mechanical properties using nanoindentation: part I. Experimental studies in an aluminum alloy. J Mater Res 11:752-759. https://doi.org/10. 1557/JMR.1996.0091

[26] Souvatzis P, Eriksson O, Katsnelson MI (2007) Anomalous thermal expansion in $\alpha$-titanium. Phys Rev Lett 99:015901. https://doi.org/10.1103/PhysRevLett.99.015901

[27] Capolungo L, Beyerlein IJ, Kaschner GC, Tomé CN (2009) On the interaction between slip dislocations and twins in HCP Zr. Mater Sci Eng A 513-514:42-51. https://doi.org/ 10.1016/j.msea.2009.01.035

[28] Fitzner A, Prakash DGL, da Fonseca JQ, Thomas M, Zhang S-Y, Kelleher J et al (2016) The effect of aluminium on twinning in binary alpha-titanium. Acta Mater 103:341-351. https://doi.org/10.1016/j.actamat.2015.09.048

Publisher's Note Springer Nature remains neutral with regard to jurisdictional claims in published maps and institutional affiliations. 